\title{
The Malleability of Attentional Capture
}

\author{
Han Zhang ${ }^{\dagger}$, Tessa R. Abagis ${ }^{\dagger}$, \& John Jonides \\ Department of Psychology, University of Michigan
}

\begin{abstract}
We suggest that consideration of trial-by-trial variations, individual differences, and training data will enrich the current framework in Luck et al. (2020). We consider whether attentional capture is modulated by trial-by-trial fluctuations of attentional state and experiences on the previous trial. We also consider whether individual differences may affect attentional capture while highlighting potential challenges in using the color-singleton task to measure individual differences. Finally, performance in the color-singleton task can be modified dramatically with practice but the underlying mechanisms are not entirely clear. Understanding the malleability of attentional capture may broaden the current framework and resolve outstanding questions.
\end{abstract}

Keywords: Attentional capture; distraction; visual search Word count: 1765

Luck and colleagues (2020) present the current state of a persistent debate: How is attention captured by salient stimuli? They outline multiple viewpoints to describe how recent literature has begun to resolve this debate and which questions still remain. In our commentary, we contribute our own perspective that examining trial-by-trial variations, individual differences, and training data may contribute to a deeper understanding of attentional capture. The common thread among our points is malleability in the control of attention it can vary from trial to trial, it can vary from person to person, and it can be improved dramatically by training. Considering mechanisms underlying this malleability will provide a more nuanced understanding of empirical data and enrich the current framework of attentional control.

First, attentional capture may be influenced by trial-by-trial fluctuations of attentional state. In Luck et al. (2020), the authors provided convincing evidence showing that participants can inhibit the salient distractor when they are given a specific search template (feature-search mode), which supports the signal suppression hypothesis. But Gaspelin and Luck (2018, p. 83) also stated that the inhibitory mechanism assumes "participants are in a state of good attentional control." As of now, it remains somewhat elusive what may constitute a "state of good attentional control." We reasoned that one possibility is that distractor suppression depends on whether

The version of record of this manuscript will be available in Visual Cognition (2021), https://doi.org/10.1080/13506285.2021. 1915903

$\dagger$ Han Zhang and Tessa R. Abagis contributed equally to this work.

Correspondence concerning this article should be addressed to Han Zhang, 530 Church Street, B434 East Hall, Ann Arbor, MI, 48109. E-mail: hanzh@umich.edu participants are engaged with the current task. Recent studies have estimated that participants spend about one-third of the time mind-wandering (having task-unrelated thoughts) during cognitive tasks (e.g., Kane et al., 2017). Mind-wandering is thought to reflect goal-neglect and has been shown to correlate with poor top-down control of oculomotor and manual responses (Mooneyham \& Schooler, 2013). Thus, momentary slips of attention may have adverse effects on distractor suppression. In a recent experiment (not yet published; Zhang, 2020), we put the idea to a test by examining whether fluctuations of task focus modulate attentional capture. Forty participants completed a "feature-search" version of the color-singleton task modeled closely after Gaspelin, Leonard, and Luck (2017)'s experiment 2. We measured attentional state in two ways. First, we presented thought probes on a subset of trials asking participants to indicate whether they were mind-wandering during the previous trial. Second, on each trial we required participants to stabilize their gaze on a cross for two seconds to trigger the search array to appear; we used gaze stability during this period to indicate the extent to which participants were ready for the upcoming search. We found that the probability of the first saccade going to the distractor did not differ between trials reported as being on-task and those reported as mindwandering (see Figure 1a). However, lower pre-trial gaze stability predicted a higher probability of having the first saccade go to the distractor on the upcoming trial (see Figure 1b). According to Luck et al. (2020), this increase in capture may be explained by weakened signal suppression of the control system or by a change in how the priority map is constructed; it is unclear whether task focus may modulate one or both factors. Overall, our preliminary results suggest a potential link between the ebbs and flows of task focus and attentional capture.

We note that task focus may not be the only factor that produces trial-by-trial variations in attentional capture. The 

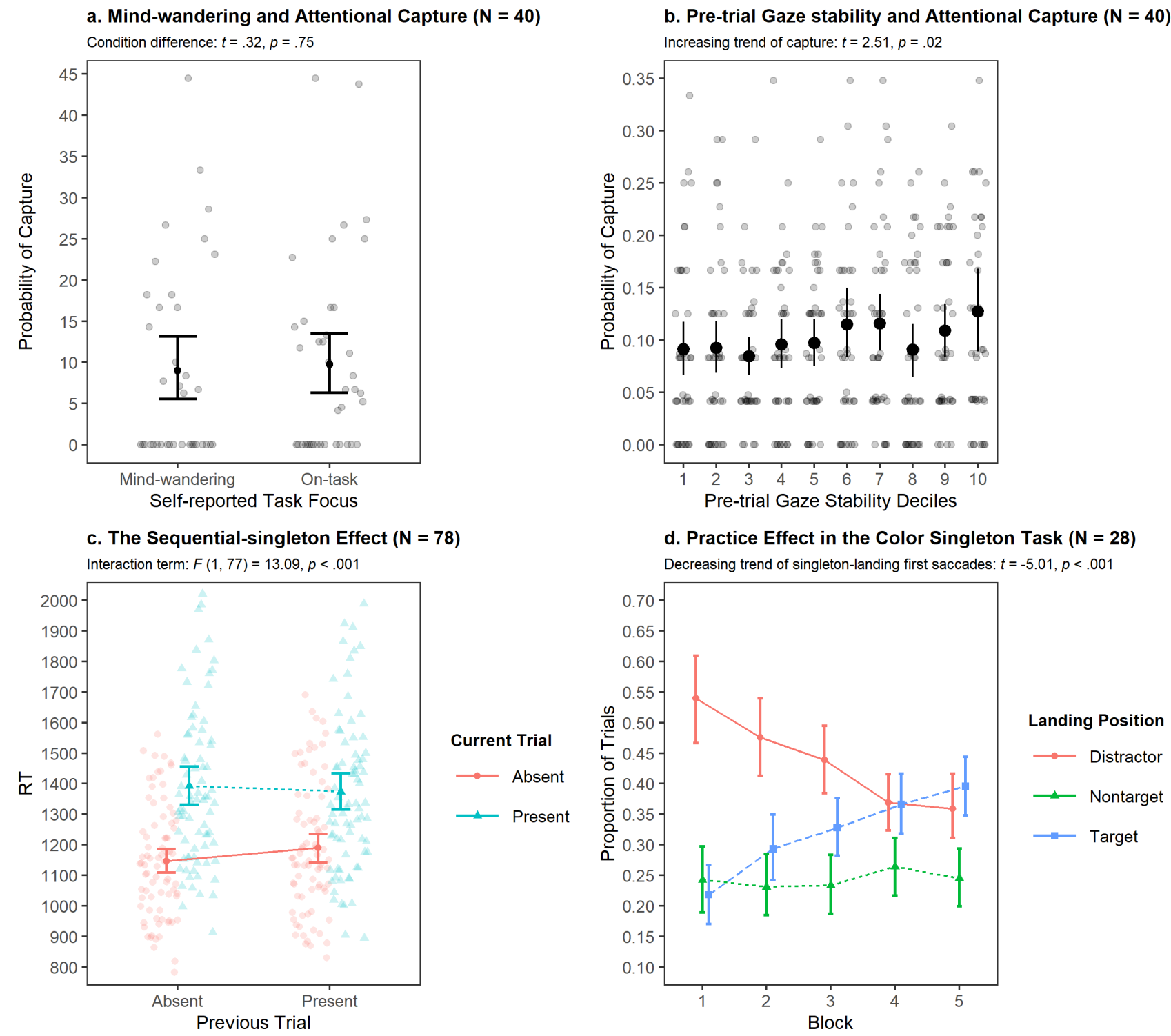

Figure 1. Panel $a$ : In a color singleton task (feature-search mode), participants did not have a higher probability of having the initial saccade landing on the color singleton if they reported they were mind-wandering compared to when they reported they were on-task (Zhang, 2020). Panel $b$ : In the same task, participants' pre-trial gaze stability significantly predicted the probability of having distractor-landing first-saccades, such that lower stability (i.e., higher deciles) predicted increased capture (Zhang, 2020). Panel $c$ : Attentional capture varied depending on whether the previous trial had a distractor or not (Abagis, 2020). Panel $d$ : Practice effect in a color singleton task where the target and the distractor features are unpredictable. In all panels, the error bars show 95\% bootstrapped confidence intervals (Abagis, 2020).

congruency-sequence effect (Weissman, Egner, Hawks, \& Link, 2015) and its perceptual counterpart, which we call here the sequential-singleton effect, occur when distractor interference is reduced following a salient, conflicting trial (such as a distractor-present trial in the color-singleton task). In a recent experiment (not yet published; Abagis, 2020), we identified a large distractor interference effect attributable to the color-singleton distractor, as expected. We also identified a sequential singleton effect such that distractor interference was reduced following a distractor-present trial (see Figure 1c), suggesting that participants may have leveraged the memory of distraction to avoid distraction on the next trial. According to Luck et al. (2020)'s framework, this attentional carryover effect from one trial to the next points to 
the role of implicit learning (selection history) in affecting subsequent performance.

We also propose that one should consider individual differences in susceptibility to attentional capture. In Luck et al. (2020), the authors disagreed on the role of working memory, with some arguing that working memory is critical to distractor suppression and others arguing that distractor suppression is solely determined by selection history (implicit learning). Indeed, we have seen evidence from both sides in the literature. For example, we know that increased WM load co-occurs with less efficient suppression of irrelevant distractors (Lavie \& De Fockert, 2005). In similar regard, Van der Stigchel (2010) found that working memory assisted in the suppression of saccades to salient, perceptual distractors. On the other hand, there is evidence that implicit learning processes (e.g., learning the likely position of the distractor) are not affected even when working memory is loaded (Gao \& Theeuwes, 2020). Most of the studies focus on examining the mean performance across individuals between conditions. But because individuals differ in their working memory and attentional control abilities, one may expect that some people are more prone to distraction than others. Given the debate highlighted in Luck et al. (2020), it may be worthwhile examining whether there are stable individual differences in attentional capture (and the ability to avoid capture) and whether they are correlated with individuals' working memory and attentional control abilities.

The challenge in examining individual differences in attentional capture is that the color-singleton task has only modest psychometric stability. The attentional capture effect is typically operationalized as a difference score in response times, and past literature has pointed out potential drawbacks of this approach (e.g., Hedge, Powell, \& Sumner, 2018). In a recent study, we recruited an online sample $(\mathrm{N}=260)$ to complete a cued version of the color-singleton task (participants were told before each trial what the target color would be). Participants completed 120 trials in total, with an equal number of distractor present and absent trials. We found a robust attentional capture effect on response times (mean difference: $222 \mathrm{~ms}, t=25.17, p<.001$, Cohen's $d=.62$ ) even though participants knew the target color in advance. Despite this large effect, the task had mediocre split-half reliability: using 5000 random splits, the Spearman-Brown corrected reliability estimate was only $0.45,95 \%$ CI $[0.33,0.55]$. So, a robust effect at the group level does not necessarily mean reliable measurement of each individual. A potential solution is to use eye-tracking measures such as initial saccade destination to measure individual differences.

Despite the potential weakness of the color-singleton task as an assay of individual differences, we did examine performance in this task and its relationship to working memory capacity in an unpublished dataset from Abagis (2020).
Participants $(\mathrm{N}=64)$ completed both the color-singleton task (singleton-detection mode) and two separate measures of working memory capacity (the automated operation span task and a visual array change detection task). We found no reliable relationship between distractor interference in the color-singleton task and either the automated operation span $(r=0.2, p=0.8)$ or visual array change detection $(r=-0.22$, $p=0.08)$. This particular result aside, we do recommend future research conducting sufficiently powered studies on individual differences in susceptibility to distraction rather than just examining mean performance across individuals.

Finally, we highlight that practice can result in improved distractor resistance. Luck et al. (2020) discussed how attentional capture can be avoided when some features of the distractor (or the target) are predictable across trials. However, attentional capture can be dramatically reduced by practice even when the features of the distractor (or the target) are completely unpredictable. In our work (not yet published; Abagis, 2020), we used eye-tracking to investigate how participants allocated their explicit attention during the colorsingleton task. The target had an equal chance to be a diamond (among circles) or a circle (among diamonds) and had an equal chance to appear in red or in green. On a random $50 \%$ of trials, the distractor appeared in a unique color. Participants completed 5 blocks of 160 trials each. We found that with repeated exposure to the task across blocks, participants were able to significantly reduce oculomotor capture. Participants were so successful at this reduction in capture that, by the final task block, they were as likely to select the target as the singleton distractor on the first saccade (see Figure 1d). Additionally, participants not only became more successful at evading the distractor, but they also became more successful at selecting the target first. These findings suggest that practice and task exposure allow participants to learn and adopt some sort of strategy to more efficiently evade the normally salient capture of a singleton distractor and isolate the target. It may be worth asking if this kind of learning is the same kind of learning in the featuresearch mode, in which participants could learn to suppress the salient distractor in a matter of a few trials. If distractor suppression in feature-search mode is primarily due to implicit learning of predictable features (as suggested in Luck et al. (2020)), one may wonder whether the practice effect with unpredictable features is simply a slower implicit learning process, or whether it stems from goal-driven control (the dotted lines around "control state" in Figure 2 of Luck et al. (2020)). Recent evidence indicates that participants are aware when they are captured by the color singleton (e.g., Adams \& Gaspelin, 2021). Perhaps this meta-cognitive knowledge about attentional capture allows participants to explicitly adjust their behavior on subsequent trials, leading to the practice effect. If indeed that is the case, it would be evidence for the role of goal-driven control in distraction 
suppression.

In sum, we expect that the debate presented by Luck et al. (2020) will remain lively. Investigations into attentional fluctuations, individual differences, and practice effects may enrich the current theoretical framework and shed light on lingering questions.

\section{References}

Abagis, T. (2020). Understanding and mitigating internal and external attention deficits in adults with ADHD (PhD thesis). University of Michigan, Ann Arbor. Retrieved from https://proxy.lib.umich.edu/login?url=https://www.proquest. com/dissertations-theses/understanding-mitigating-internalexternal/docview/2480291804/se-2 ?accountid=14667

Adams, O. J., \& Gaspelin, N. (2021). Introspective awareness of oculomotor attentional capture. Journal of Experimental Psychology: Human Perception and Performance.

Gao, Y., \& Theeuwes, J. (2020). Learning to suppress a distractor is not affected by working memory load. Psychonomic Bulletin \& Review, 27(1), 96-104.

Gaspelin, N., Leonard, C. J., \& Luck, S. J. (2017). Suppression of overt attentional capture by salient-but-irrelevant color singletons. Attention, Perception, \& Psychophysics, 79(1), 45-62. https://doi.org/10.3758/s13414-016-1209-1

Gaspelin, N., \& Luck, S. J. (2018). The role of inhibition in avoiding distraction by salient stimuli. Trends in Cognitive Sciences, 22(1), 79-92. https://doi.org/10.1016/j.tics.2017. 11.001

Hedge, C., Powell, G., \& Sumner, P. (2018). The reliability paradox: Why robust cognitive tasks do not produce reliable individual differences. Behavior Research Methods, 50(3), 1166-1186.

Kane, M. J., Gross, G. M., Chun, C. A., Smeekens, B. A., Meier, M. E., Silvia, P. J., \& Kwapil, T. R. (2017). For whom the mind wanders, and when, varies across laboratory and daily-life settings. Psychological Science, 28(9), 12711289. https://doi.org/10.1177/0956797617706086

Lavie, N., \& De Fockert, J. (2005). The role of working memory in attentional capture. Psychonomic Bulletin \& Review, 12(4), 669-674. https://doi.org/10.3758/BF03196756

Luck, S. J., Gaspelin, N., Folk, C. L., Remington, R. W., \& Theeuwes, J. (2020). Progress toward resolving the attentional capture debate. Visual Cognition, 1-21. https: //doi.org/10.1080/13506285.2020.1848949

Mooneyham, B. W., \& Schooler, J. W. (2013). The costs and benefits of mind-wandering: A review. Canadian Journal of Experimental Psychology/Revue Canadienne de Psychologie Expérimentale, 67(1), 11-18. https://doi.org/10.1037/ a0031569

Van der Stigchel, S. (2010). The search for oculomotor inhibition: Interactions with working memory. Experimental Psychology, 57(6), 429.

Weissman, D. H., Egner, T., Hawks, Z., \& Link, J. (2015). The congruency sequence effect emerges when the distracter precedes the target. Acta Psychologica, 156, 8-21.

Zhang, H. (2020). Mind-wandering: What can we learn from eye movements? ( $\mathrm{PhD}$ thesis). University of Michigan, Ann Arbor. Retrieved from https://proxy.lib.umich.edu/login?url=https://www. proquest.com/dissertations-theses/mind-wandering-whatcan-we-learn-eye-movements/docview/2405343785/se2 ? accountid $=14667$ 